\title{
Analyzing the symmetrical arrangement of structural repeats in proteins with CE-Symm [S4 Tab]
}

Spencer E Bliven, Aleix Lafita, Peter W Rose, Guido Capitani, Andreas Prlić, Philip E Bourne

\begin{tabular}{ccc}
\hline Method & Precision & Cramer V \\
\hline GraPhCOMPONENT & 0.598 & 0.652 \\
DeLTAPosition & 0.783 & 0.728 \\
RotATIONANGLE & 0.754 & 0.642 \\
\hline
\end{tabular}

S4 Tab. Performance measures of the symmetry order detection methods for domains in the benchmark dataset with closed symmetry. Precision measures the total fraction of correct predictions and Cramer V measures the correlation between actual and predicted classes. Both measures have values in the $[0,1]$ interval, where 1 means perfect precision and correlation. 